\title{
Palinología de Agavaceae, una contribución biosistemática
}

\author{
LINA OJEDA REVAH ${ }^{1}$ Y BEATRIZ LUDLOW WIECHERS ${ }^{2}$
}

'El Colegio de la Frontera Norte, Blvd. Abelardo L. Rodríguez 21, Zona del Río, Tijuana, Baja California, México. ${ }^{2}$ Laboratorio de Plantas Vasculares, Facultad de Ciencias, UNAM, México, D.F.

\begin{abstract}
Resumen. Se estudia el polen de 15 géneros y 36 especies de la familia Agavaceae según la clasificación de Hutchinson. Las muestras fueron observadas al MEB y ML. Se revisan diferentes clasificaciones taxonómicas considerando la morfología del polen, distribución geográfica, cariotipo y posición del ovario. Desde el punto de vista palinológico, la última clasificación de Dahlgren et al.; coincide con los resultados obtenidos. Las muestras de polen estudiadas presentan dos niveles de comportamiento morfológico; en el nivel interespecífico la variación encontrada es mínima o está ausente y en el nivel intergenérico se presentan los siguientes patrones morfológicos: a) semitectado y principalmente monosulcado: Agave, Beschorneria, Dasylirion, Furcraea, Hesperaloë y Manfreda. En el caso de Polianthes a veces se encuentra disulcado (Agavaceae, Agavoideae); b) tectado perforado y monosulcado: Yucca (Agavaceae, Yuccoideae) y Dracaena americana (Dracaenaceae); c) tectado perforado a microrreticulado y monosulcado a disulcado: Beaucarnea, Calibanus y Nolina (Nolinaceae); d) intectado verrugado y monosulcado: Hosta (Funkiaceae); e) semitectado a tectado perforado y tricotomosulcado: Phormium (Phormiaceae); f) fosulado y monosulcado: Cordyline (Asteliaceae) y Dracaena fragans (Dracaenaceae) y g) psilado y ulcerado: Sansevieria (Dracaenaceae).

Palabras clave: Agavaceae, cariotipo, fitogeografía, morfología, polen, sistemática.
\end{abstract}

\begin{abstract}
The pollen of 15 genera and 36 species of the family Agavaceae were studied, based on Hutchinson's classification. Observations were made with LM and SEM. Different taxonomical classifications are reviewed considering pollen morphology, geographical distribution, chromosome number and ovary position. From palynological point of view, Dahlgren et al., last classification agrees with the results obtained. Pollen samples studied show two levels of morphological behaviour: at an interespecific level there is little or no variation and at a generic level the following patterns are observed: a) semitectate and mainly monosulcate: Agave, Beschorneria, Dasylirion, Furcraea, Hesperaloë Manfreda and Polianthes sometimes disulcate (Agavaceae, Agavoideae); b) tectate-perforate and monosulcate: Yucca (Agavaceae, Yuccoideae) and Dracaena americana (Dracaenaceae); c) tectate perforate to microreticulate and monosulcate to disulcate: Beaucarnea, Calibanis and Nolina (Nolinaceae); d) intectate verrugate and monosulcate: Hosta (Funkiaceae); e) semitectate to tectate perforate and trichotomosulcate: Phormium (Phormiaceae); f) fosulate and monosu cate: Cordyline (Asteliaceae) and Dracaena fragans (Dracaenaceae) and g) psilate and ulcerate: Sansevieria (Dracaenaceae).
\end{abstract}

Key words: Agavaceae, karyotype, morphology, phytogeography, pollen, systematics.

\section{INTRODUCCIÓN}

La familia Agavaceae, de gran importancia en México, está constituida por especies de las cuales se obtienen numerosos productos, entre los que destacan las fibras naturales y las bebidas alcohólicas. Además, la mayoría de los géneros son ornamentales en casi todo el mundo (Verhoek, 1978).

Los miembros de esta familia (sensu Traub, 1953), se distribuyen en los trópicos y subtrópicos del mundo, especialmente en las regiones áridas y semiáridas. En México y el sur de Estados Unidos crecen los géneros Agave, Dasylirion, Hesperaloë, Manfreda, Nolina y Yucca. Con una distribución restringida a México están: Beschorneria, Beaucarnea, Furcraea, Polianthes y Prochnyanthes.

García-Mendoza (1995) realiza un análisis areográfico de 198 especies de Agavaceae mexicanas, incluidas en ocho géneros, y encontró que México es el centro de mayor riqueza y diversidad de especies, ya que en el país se localizan el $75 \%$ del total de especies, de las cuales un 55\% de ellas son endémicas.

El resto de los miembros de la familia se distribuyen hacia el Viejo Mundo. Dracaena se encuentra en Africa subtropical y Asia, hasta el norte de Australia; mientras que Hosta habita en China y Japón; Phormium en Nueva Zelandia; Sansevieria en Africa subtropical, sur de Madagascar y Arabia; Doryanthes en Australia; Cohnia en las islas Mascareñas y Nueva Caledonia y Cordyline, en Indomalasia, Nueva Zelandia, Polinesia, Hawaii, Australia, Africa y Sudamérica.

Desde el punto de vista taxonómico, la familia Agavaceae ha sido objeto de amplias revisiones, ya que desde su creación por Endlicher (1841, en Gómez Pompa et al., 1971) ha sido muy discutida. Por un lado, algunos autores como Bentham y Hooker (1883), Millspaugh (1898), Trelease (1920), Pax y Hoffman (1930), Lundell (1937), Conzatti (1946) y Matuda $(1960,1962)$, entre otros, no la han reconocido como familia y consideran a sus géneros como parte de 
Liliaceae o de Amaryllidaceae. Por otro lado, a partir de una propuesta de Hutchinson (1934), Traub (1953) publica una clave para tribus y géneros de Agavaceae, criterio que posteriormente es utilizado por Standley (1930), Berlin (1953), Howard (1979) y Wiggins (1980), entre otros.

Estudios cariológicos como los de McKelvey y Sax (1933), Whitaker (1934), Satô (1935), Vignoli (1936, 1937), Granick (1944), Sharma y Battacharyya (1962), Sharma y Chaudhuri (1964), Gómez-Pompa et al. (1971), han servido para separar algunos géneros de la familia propuesta por Hutchinson (1934) y situarlos en otras o formar grupos genéricos de diferente nivel taxonómico dentro de Agavaceae (Cronquist, 1968; Takhtajan, 1980; Dahlgren y Clifford, 1982; Dahlgren et al., 1985).

En cuanto a los trabajos palinológicos realizados en esta familia, existe un gran número de ellos. En floras palinológicas en las que se mencionan algunos géneros o especies están los trabajos de Erdtman (1952), Cranwell (1953), Tsukada (1964), Sharma (1967), González-Quintero (1969), Martínez-Hernández (1970), Bonefille (1971), Heusser (1971), Huang (1972) y Markgraff y D’Antoni (1978). En revisiones de géneros o especies, se encuentran los estudios de Palacios-Chávez (1974, 1978), Ludlow-Wiechers y Ojeda (1983), García-Mendoza (1987) y Palacios-Chávez et al. (1988). Radulescu (1973), Dahlgren y Clifford (1982) y Zavada (1983) describen el polen a nivel de familia y de orden.

Johnson y Gale (1983) realizan un estudio sistemático en tres especies diferentes de Calibanus, Agave y Dasylirion, comparando la morfología de la hoja, cariotipo y polen.

En trabajos mas detallados, Ojeda et al. (1984) estudian el polen de diez especies de seis géneros de Agavaceae, y encontraron que en función del polen resulta difícil mantener a la familia tal y como la propuso Hutchinson (1934), aproximándose mejor a la propuesta de Takhtajan (1980). Por su parte, Álvarez y Köhler (1987) examinan el polen de 16 géneros presentes en América, además de Hosta, de los cuales cinco se encuentran actualmente en sinonimia. Estos autores, basados en la asociación, número y tipo de abertura, caracteres del margen y exina, reconocen ocho tipos de polen: tipo Yucca; tipo Agave (subtipo Agave-Manfreda, subtipo Bravoa-Prochnyanthes, subtipo BeschorneriaFurcraea, subtipo Hesperaloë); tipo Hosta; tipo Hosta plantaginea; tipo Hesperocallis; tipo Leucocrinum y tipo Manfreda singuliflora.

El presente trabajo se basó en la clasificación de Traub (1953 y 1972), quien agrega Hosta a la clasificación de Hutchinson (1934). Sin embargo, Doryanthes, Cohnia, y Prochnyanthes no se incluyeron por no haber ejemplares de herbario con estructuras florales suficientes para su observación. El género Calibanus es considerado a través del trabajo de Johnson y Gale (1983).

\section{MATERIALES Y MÉTODO}

Las muestras fueron obtenidas de los ejemplares depositados en los herbarios CHAPA, MEXU y ENCB (Cuadro 1). Se dividieron en dos grupos: en el primero se encuentran las muestras no acetolizadas y observadas al microscopio de barrido, las cuales fueron sombreadas y cubiertas con $\mathrm{Au}$ y posteriormente introducidas en un microscopio JEOL, modelo JSMT-20, fotografiadas en placa Royal Pan 400 ASA e impresas por contacto en papel Kodabromide F3.

En el segundo grupo se encuentran las muestras acetolizadas y estudiadas al microscopio de luz. La acetólisis se realizó siguiendo la técnica de Erdtman (1943), previo tratamiento con $\mathrm{KOH}$ al $10 \%$ y los granos de polen se montaron en gelatina glicerinada. Las medidas se hicieron en diez granos de polen tomados al azar. Para las fotografías se utilizó un microscopio Zeiss standar 7, con cámara adaptada y película Plus $\mathrm{X}$ pan y fueron impresas en papel Kodabromide F3 y F5.

Las colecciones de las laminillas para microscopio de luz (ML) y fotografías al microscopio electrónico de barrido (MEB) -material de referencia para este estudio- se encuentran en el Herbario MEXU.

En relación con las descripciones morfológicas, la terminología usada está basada en Erdtman (1952), en la enciclopedia palinológica de Kremp (1965) y en Praglowski y Punt (1973).

Para obtener la forma de los granos de polen, debido a que las aberturas son polares distales, no se ha considerado la relación $(\mathrm{P} / \mathrm{E})$, utilizada para dicotiledóneas, que significa el eje polar entre el eje ecuatorial, en vista ecuatorial. En su lugar, se consideran los ejes ecuatorial mayor y ecuatorial menor, en vista polar. Cabe mencionar que en trabajos anteriores, Ludlow-Wiechers y Ojeda (1983) y Ojeda et al. (1984) han seguido este criterio; sin embargo, al indicar la forma no se han puntualizado los ejes considerados.

\section{RESULTADOS}

\section{Descripciones palinológicas}

Las medidas de los ejes y la exina se encuentran en los Cuadros 2, 3 y 4 . El arreglo de las microfotografías en las láminas sigue una secuencia alfabética de los géneros observados y no están organizadas considerando las clasificaciones taxonómicas revisadas.

\section{Beaucarnea inermis (S. Watson) Rose}

(Lámina I, Figs. 1 a 3)

Eumónada, heteropolar, bilateral; monosulcada y disulcada, sulco tan largo como el eje ecuatorial mayor en granos monosulcados; ornamentación de la exina al MEB y ML tectada perforada; elíptico.

\section{Beaucarnea stricta Lem. \\ (Lámina I, Figs. 4 a 6).}

Eumónada, heteropolar, bilateral; monosulcada, sulco tan largo como el eje ecuatorial mayor y con terminaciones desiguales; ornamentación de la exina al MEB tectada perforada y en la zona del polo proximal microrreticulada; al ML microrreticulada; elíptico.

Beschorneria yuccoides $\mathrm{K}$. Koch

(Lámina I, Figs. 7 a 9). 
Cuadro 1. Ejemplares revisados para la familia Agavaceae (sensu Traub, 1953).

Especie

Beaucarnea inermis ( $S$. Watson) Rose

Beaucarnea stricta Lem.

Beschorneria yuccoides K. Koch

Dasylirion acrotriche Zucc.

Dasylirion cedrosanum Trel.

Furcraea bedinghausii K. Koch

Furcraea guatemalensis Trel.

Hesperaloë funifera (K. Koch) Trel.

Hesperaloë parviflora (Torr.) Coult.

Hosta sieboldiana (Lodd.) Engl.

Manfreda scabra (Ort.) McVaugh

Manfreda brunnea (S. Watson) Rose

Manfreda pringlei Rose

Manfreda singuliflora ( $S$. Watson) Rose

Nolina nelsonii Rose

Nolina parviflora (HBK) Hemsl.

Phormium tenax Forster et G. Foster

Polianthes geminiflora La Llave et Lex.

Polianthes pringlei Rose

Yucca torreyi Shafer
Colector

Castillo

Trigos

Hernández

García

Wendt y Riskin

Matuda

Molina

Johnston, Wendt, Chiang

Matuda

Yamazaki

Galván

Chiang

Galván

Johnston

Chiang

Ramos

Koch

Hernández

Matuda

Matuda
No. de colecta

146

529

4130

328

1694

37561

26518

10892

38791

7048

1239

12298

890

8542

11146

57

7621

4556

29250

38560
Herbario

M.EXU

MEXU

MEXU

CHAPA

CHAPA

MEXU

ENCB

MEXU

ENCB

MEXU

ENCB

MEXU

ENCB

MEXU

MEXU

MEXU

MEXU

MEXU

MEXU

ENCB
Tétrada tetragonal, heteropolar, bilateral; inaperturada; ornamentación de la exina al MEB y ML semitectada, con muros discontinuos, heterobrocada, lúmenes de 2.5 a 10.5 $\mu \mathrm{m}$ y muros de 1.8 a $3.5 \mu \mathrm{m}$, simplibaculada, a veces duplibaculada, con columelas en los lúmenes; elíptico.

Dasylirion acrotriche Zucc.

(Lámina II, Figs. 1 a 5)

Eumónada, heteropolar, bilateral; monosulcada, sulco tan largo como el eje ecuatorial mayor; ornamentación de la exina al MEB semitectada, al ML semitectada y microrreticulada en la zona de la abertura, heterobrocada, lúmenes de 1 a 2.5 $\mu \mathrm{m}$ y muros $0.8 \mu \mathrm{m}$, simplibaculada a duplibaculada; elíptico.

Dasylirion cedrosanum Trel.

(Lámina II, Figs. 6 a 8)

Eumónada, heteropolar, bilateral; monosulcada, sulco tan largo como el eje ecuatorial mayor, ornamentación de la exina al ML de microrreticulada a semitectada en el polo proximal, heterobrocada, lúmenes de 0.8 a $3 \mu \mathrm{m}$ y muros de $0.8 \mu \mathrm{m}$, simplibaculada a duplibaculada; elíptico.

\section{Furcraea bedinghausii $\mathrm{K}$. Koch}

(Lámina III, Figs. 1 a 6)

Tétrada tetragonal, heteropolar, bilateral; inaperturada; ornamentación de la exina al MEB y ML en la zona polar distal semitectada y en el resto de la exina tectada perforada, heterobrocada, lúmenes de 1.5 a $7 \mu \mathrm{m}$ y muros de 1 a $3 \mu \mathrm{m}$, simplibaculada; elíptico.

Furcraea guatemalensis Trel.

(Lámina III, Figs. 7 a 10)

Tétrada tetragonal, heteropolar, bilateral; monosulcada, sulco al MEB con membrana y restos de la exina y al ML se desprende quedando los márgenes del sulco mal definidos; ornamentación de la exina al MEB y al ML de tectada perforada a semitectada, retículo presente en la zona de la abertura, heterobrocada, lúmenes de 1 a $4 \mu \mathrm{m}$ y muros de 3 a $5.5 \mu \mathrm{m}$, simplibaculada; elíptico.

Hesperaloë funifera (K. Koch) Trel.

(Lámina IV, Fig .1)

Eumónada, heteropolar, bilateral; monosulcada, sulco tan largo como el eje ecuatorial mayor, ornamentación de la exina al MEB y ML semitectada, heterobrocada, simplibaculada a duplibaculada; elíptico.

Hesperaloë parviflora (Torr.) Coult.

(Lámina IV, Figs. 2 a 5)

Eumónada, heteropolar, bilateral; monosulcada, sulco tan largo como el eje ecuatorial mayor, ornamentación de la exina al MEB y ML semitectada, heterobrocada, lúmenes de 1 a $3 \mu \mathrm{m}$ y muros de 0.8 a $1 \mu \mathrm{m}$, simplibaculada a duplibaculada; elíptico. 
CUADRo 2. Medidas de los ejes y exina para el polen de las especies de Agavaceae estudiadas (sensu Traub 1953).

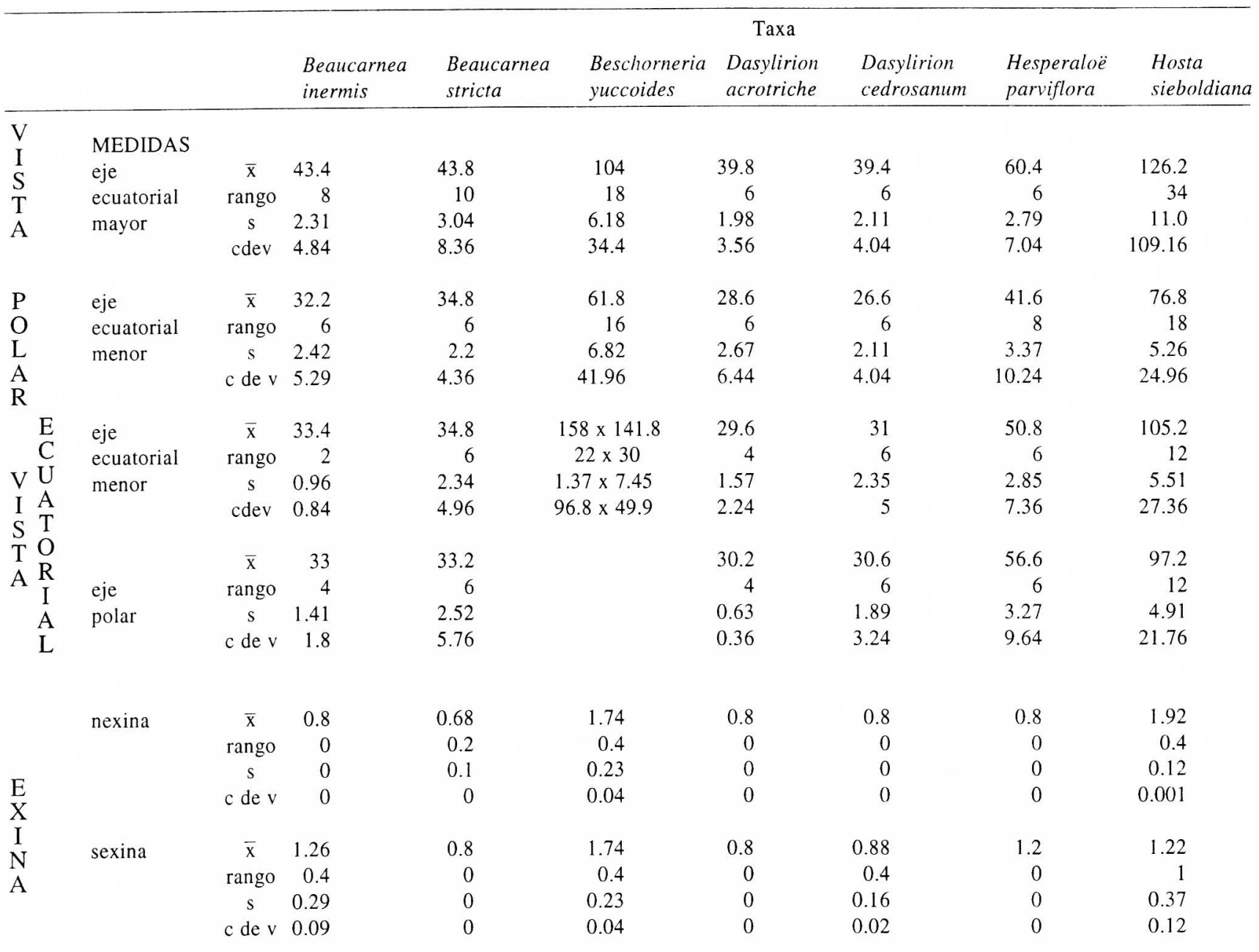

Hosta sieboldiana (Lodd.) Engl.

(Lámina IV, Figs. 6 a 9)

Eumónada, heteropolar, bilateral, monosulcada, ornamentación de la exina al MEB y ML intectada, verrugada a gemada; elíptico.

Manfreda brunnea (S. Watson) Rose

(Lámina V, Figs. 2 y 3 )

Eumónada, heteropolar, bilateral; monosulcada, sulco de ca. $98.5 \mu \mathrm{m}$; ornamentación de la exina al MEB y ML semitectada, al MEB tectada perforada en la zona de la abertura, heterobrocada, lúmenes de 2 a $8 \mu \mathrm{m}$ y muros de 1.5 a $8 \mu \mathrm{m}$, multibaculada, con columelas libres en los lúmenes de menos de $1 \mu \mathrm{m}$; elíptico.

Manfreda pringlei Rose

(Lámina V, Figs. 4 a 7)

Eumónada, heteropolar, bilateral; monosulcada, sulco tan largo como el eje ecuatorial mayor; ornamentación de la exina al MEB y ML semitectada, heterobrocada, lúmenes de 2 a $12 \mu \mathrm{m}$ y muros de 1 a $1.5 \mu \mathrm{m}$, simplibaculada a duplibaculada; elíptico.

Manfreda scabra (Ort.) McVaugh (Lámina V, Fig. 1)

Eumónada, heteropolar, bilateral; monosulcada, sulco de ca. $85 \mu \mathrm{m}$; ornamentación de la exina al MEB y ML semitectada, heterobrocada, lúmenes de 2 a $14 \mu \mathrm{m}$ y muros de 2 a $3 \mu \mathrm{m}$, simplibaculada escasamente duplibaculada, columelas de 1 $\mu \mathrm{m}$; elíptico.

Manfreda singuliflora (S. Watson) Rose

(Lámina V, Figs. 8 y 9)

Eumónada, heteropolar, bilateral; monosulcada, sulco de ca. $85 \mu \mathrm{m}$; ornamentación de la exina al MEB y ML semitectada y en la zona cercana al sulco microrreticulada, heterobrocada, lúmenes de 2 a $11.5 \mu \mathrm{m}$ y muros de 2 a $3 \mu \mathrm{m}$, simplibaculada, 
CuAdro 3. Medidas de los ejes y exina para el polen de las especies de Agavaceae estudiadas (sensu Traub 1953).

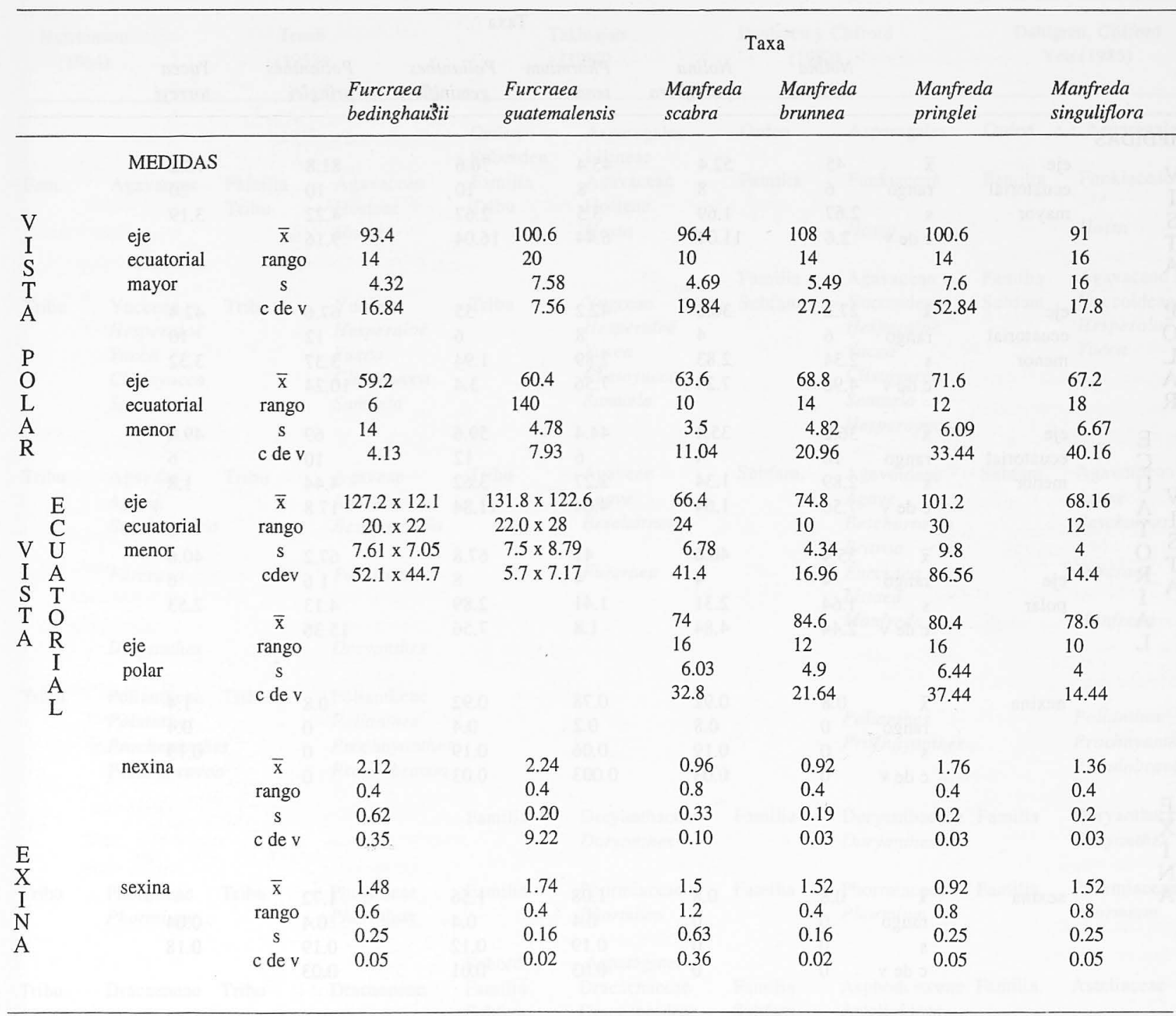

con columelas libres en los lúmenes de ca. $2 \mu \mathrm{m}$; elíptico. Nolina nelsonii Rose

(no hay figuras)

Eumónada, heteropolar, bilateral; monosulcada, sulco tan largo como el eje ecuatorial mayor; ornamentación de la exina al ML microrreticulada, heterobrocada, lúmenes de 1 a $1.5 \mu \mathrm{m}$ y muros de $0.8 \mu \mathrm{m}$ en la zona de la abertura, microrretículo más pequeño, simplibaculada; elíptico.

Nolina parviflora (HBK) Hemsl.

(Lámina VI, Figs. 1 y 2)

Eumónada, heteropolar, bilateral; monosulcada, sulco de ca.46um;ornamentación de laexinaal MEB yML, microrreticulada, con lúmenes hasta de $1.6 \mu \mathrm{m}$, heterobrocada; elíptico.

Phormium tenax Forster et G. Forster

(Lámina VI, Figs. 3 a 6)
Eumónada, heteropolar, radial; tricotomosulcada, al MEB con membrana de la nexina muy evidente; ornamentación de la exina al MEB y ML semitectada en el polo proximal y haciéndose microrreticulada hasta tener un margo tectado perforado, lúmenes menores de $1 \mathrm{a} 4 \mu \mathrm{m}$ y muros de 0.8 a $1.6 \mu \mathrm{m}$, simplibaculada a duplibaculada; triangular.

Polianthes geminiflora La Llave et Lex.

(Lámina VII, Figs. 1 a 4)

Eumónada, heteropolar, bilateral; monosulcada o disulcada, sulco de ca. $71.5 \mu \mathrm{m}$, sin acetolizar con membrana de la nexina muy evidente; ornamentación de la exina al MEB y ML semitectada y algunas veces microrreticulada en la zona de la abertura, heterobrocada, lúmenes de 1.5 a $6 \mu$ m y muros de 0.8 a $2 \mu \mathrm{m}$, simplibaculada con columelas de ca. $0.8 \mu \mathrm{m}$; elíptico.

Polianthes pringlei Rose

(Lámina VII, Figs. 5 a 10) 
Cuadro 4. Medidas de los ejes y exina para el polen de las especies de Agavaceae estudiadas (sensu Traub 1953).

Taxa

$\begin{array}{llllll}\text { Nolina } & \text { Nolina } & \text { Phormium } & \text { Polianthes } & \text { Polianthes } & \text { Yucca } \\ \text { nelsonii } & \text { parviflora } & \text { tenax } & \text { geminiflora } & \text { pringlei } & \text { torreyi }\end{array}$

\begin{tabular}{|c|c|c|c|c|c|c|c|c|}
\hline \multicolumn{9}{|c|}{ MEDIDAS } \\
\hline \multirow{5}{*}{$\begin{array}{l}\mathrm{V} \\
\mathrm{I} \\
\mathrm{S} \\
\mathrm{T} \\
\mathrm{A}\end{array}$} & \multirow{5}{*}{$\begin{array}{l}\text { eje } \\
\text { ecuatorial } \\
\text { mayor }\end{array}$} & $\bar{x}$ & 45 & 52.4 & 45.4 & 76.6 & 81.8 & 78.2 \\
\hline & & rango & 6 & 8 & 8 & 10 & 10 & 20 \\
\hline & & s & 2.67 & 1.69 & 3.5 & 2.67 & 4.22 & 3.19 \\
\hline & & $\mathrm{cde} \mathrm{v}$ & 2.6 & 11.04 & 6.44 & 16.04 & 9.16 & \\
\hline & & & & & & & & \\
\hline \multirow{4}{*}{$\begin{array}{l}\mathrm{P} \\
\mathrm{O} \\
\mathrm{L} \\
\mathrm{A} \\
\mathrm{R}\end{array}$} & \multirow{4}{*}{$\begin{array}{l}\text { eje } \\
\text { ecuatorial } \\
\text { menor }\end{array}$} & $\bar{x}$ & 27.2 & 30.6 & 42.2 & 55 & 67.6 & 42.4 \\
\hline & & rango & 6 & 4 & 8 & 6 & 12 & 10 \\
\hline & & s & 2.34 & 2.83 & 2.89 & 1.94 & 3.37 & 3.32 \\
\hline & & $c$ de $v$ & 4.96 & 7.24 & 7.56 & 3.4 & 10.24 & \\
\hline \multirow{8}{*}{$\begin{array}{cc} & \mathrm{E} \\
& \mathrm{C} \\
\mathrm{V} & \mathrm{U} \\
\mathrm{V} & \mathrm{A} \\
\mathrm{I} & \mathrm{T} \\
\mathrm{S} & \mathrm{O} \\
\mathrm{T} & \mathrm{R} \\
\mathrm{A} & \mathrm{I} \\
& \mathrm{A} \\
& \mathrm{L}\end{array}$} & \multirow{4}{*}{$\begin{array}{l}\text { eje } \\
\text { ecuatorial } \\
\text { menor }\end{array}$} & $\bar{x}$ & 36.2 & 35.4 & 44.4 & 59.6 & 69 & 49.4 \\
\hline & & rango & 10 & 4 & 6 & 12 & 10 & 6 \\
\hline & & s & 2.89 & 1.34 & 2.27 & 3.62 & 4.44 & 1.8 \\
\hline & & $\mathrm{c}$ de $\mathrm{v}$ & 7.56 & 1.64 & 4.64 & 11.84 & 17.8 & \\
\hline & \multirow{4}{*}{$\begin{array}{l}\text { eje } \\
\text { polar }\end{array}$} & $\bar{x}$ & 35.4 & 40.6 & 41 & 67.8 & 672 & 406 \\
\hline & & rango & 4 & 4 & $\begin{array}{r}1 \\
4\end{array}$ & $\begin{array}{r}1.0 \\
8\end{array}$ & $\begin{array}{r}07.2 \\
16\end{array}$ & $\begin{array}{r}40.0 \\
6\end{array}$ \\
\hline & & s & 1.64 & 2.31 & 1.41 & 2.89 & 4.13 & 2.53 \\
\hline & & $\mathrm{c}$ de $\mathrm{v}$ & 2.44 & 4.84 & 1.8 & 7.56 & 15.36 & \\
\hline \multirow{7}{*}{$\begin{array}{c}E \\
X \\
I \\
N\end{array}$} & \multirow[t]{4}{*}{ nexina } & $\overline{\mathrm{x}}$ & 0.8 & 0.92 & 0.78 & 0.92 & 0.8 & 1.4 \\
\hline & & rango & 0 & 0.8 & 0.2 & 0.4 & 0 & 0.4 \\
\hline & & s & 0 & 0.19 & 0.06 & 0.19 & 0 & 0.19 \\
\hline & & $c$ de $v$ & 0 & 0.03 & 0.003 & 0.03 & 0 & \\
\hline & & & & & & & & \\
\hline & & & & & & & & \\
\hline & & & & & & & & \\
\hline \multirow[t]{4}{*}{ A } & \multirow[t]{4}{*}{ sexina } & $\overline{\mathrm{x}}$ & 0.8 & 0.8 & 1.08 & 1.56 & 1.72 & \\
\hline & & rango & 0 & 0 & 0.4 & 0.4 & 0.4 & 0.04 \\
\hline & & $\mathrm{s}$ & 0 & 0 & 0.19 & 0.12 & 0.19 & 0.18 \\
\hline & & $\mathrm{c}$ de $\mathrm{v}$ & 0 & 0 & 0.03 & 0.01 & 0.03 & \\
\hline
\end{tabular}

Eumónada, heteropolar, bilateral; monosulcada y disulcada, la abertura tan larga como el eje ecuatorial mayor; ornamentación de la exina al MEB y ML semitectada y en la zona de la abertura microrreticulada, heterobrocada, lúmenes de 1 a $4 \mu$ m y muros de 1 a $2 \mu \mathrm{m}$, simplibaculada; elíptico.

Yuca torreyi Shafer

\section{(Lámina VI, Figs. 7 y 8)}

Eumónada, heteropolar, bilateral; monosulcada, sulco de ca. 65.6 $\mu \mathrm{m}$; ornamentación de la exina al ML puntitegilada; elíptico.

\section{DISCUSIÓN Y CONCLUSIONES}

Como se mencionó al principo, la familia Agavaceae ha presentado muchos problemas en su delimitación genérica. Esto se debe en gran parte a la complejidad del grupo y a la inclusión de géneros que han sido considerados sinónimos por algunos autores. Las clasificaciones propuestas por Hutchinson (1964), Traub (1953, 1972), Takhtajan (1980) y Dahlgren y Clifford (1982) presentan algunos de estos problemas que posteriormente son considerados por Dahlgren et al. (1985) (Cuadro 5).Los géneros Clistoyucca, Hesperoyucca y Samuela, originalmente considerados independientes, son fusionados con Yucca por McKelvey (1938-1947), criterio que después fue apoyado por Matuda y Piña (1980), quedando la subfamilia Yuccoideae (Dahlgren et al., 1985), formada exclusivamente por Hesperaloë y Yucca.

El género Pseudobravoa, omitido por Takhtajan (1980), se considera actualmente sinónimo de Polianthes, aunque por otra parte se reconoce como subgénero de este último (Verhoek, 1975). 
Cuadro 5. Clasificaciones taxonómicas de Agavaceae y familias relacionadas.

$\begin{array}{ccccc}\text { Hutchinson } & \text { Traub } & \text { Takhtajan } & \text { Dahlgren y Clifford } & \text { Dahlgren, Clifford } \\ (1964) & (1953) & (1980) & (1982) & \text { Yeo (1985) }\end{array}$

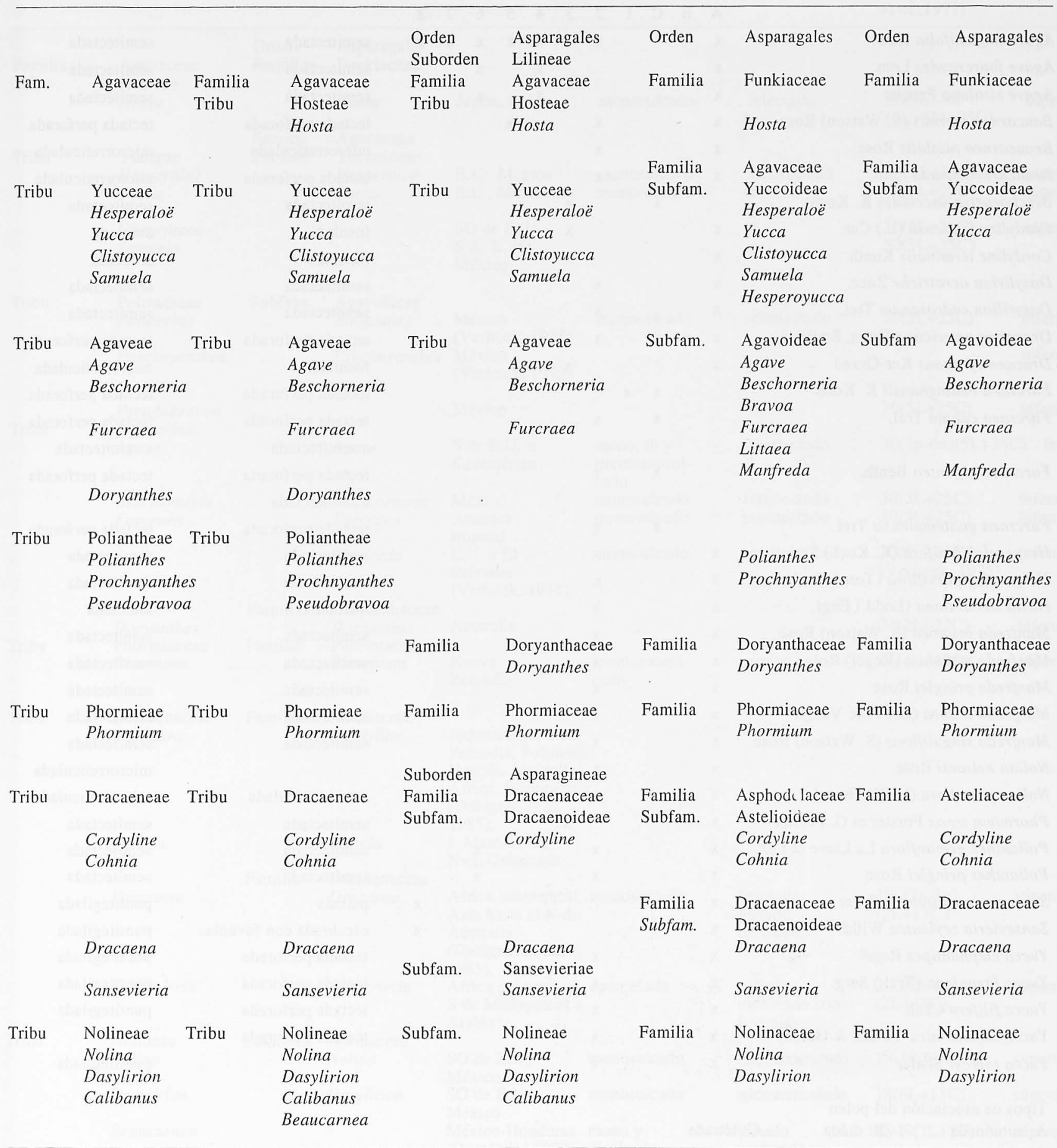

En la tribu Nolineae, constituida por Nolina, Dasylirion y Calibanus, el género Beaucarnea es omitido siempre, excepto por Traub (1953) y Matuda y Piña (1980). Dahlgren et al. (1985) elevan esta tribu a nivel de familia, formada por tres o cuatro géneros, mencionando sólo a Nolina y a Dasylirion. Sin embargo, en este trabajo, se considera a 
CuAdro 6. Asociación, abertura y ornamentación de especies de Agavaceae tratadas en este trabajo, Ludlow-Wiechers et al. (1984) y Ojeda et al. (1986) ESPECIE

Agave angustifolia Haw.

Agave fourcroydes Lem.

Agave sisalana Perrine

Baucarnea inermis (S. Watson) Rose

Beaucarnea pliabilis Rose

Beaucarnea stricta Lem.

Beschorneria yuccoides K. Koch

Cordyline fruticosa (L.) Car.

Cordyline terminalis Kunth

Dasylirion acrotriche Zucc.

Dasyrilion cedrosanum Trel.

Dracaena americana Donn. Smith

Dracaena fragans Ker-Grawl

Furcraea bedinghausii K. Koch

Furcraea cahum Trel.

Furcraea gigantea Benth.

Furcraea guatemalensis Trel.

Hesperaloë funifera (K. Koch) Trel.

Hesperaloë parviflora (Torr.) Coult.

Hosta sieboldiana (Lodd.) Engl.

Manfreda brunnea (S. Watson) Rose

Manfreda maculata (Regel) Rose

Manfreda pringlei Rose

Manfreda scabra (Ort.) Mc Vaugh

Manfreda singuliflora (S. Watson) Rose

Nolina nelsonii Rose

Nolina parviflora (HBK) Hemsl.

Phormium tenax Forster et G. Forster

Polianthes geminiflora La Llave et Lex.

Polianthes pringlei Rose

Sansevieria cylindrica Bojer

Sansevieria zeylanica Willd.

Yucca elephantipes Regel

Yucca faxoniana (Trel.) Sarg.

Yucca filifera Chab.

Yucca lacandonica Valdéz \& Gómez

Yucca torreyi Shafer

\begin{tabular}{|c|c|c|c|c|c|c|c|}
\hline A & B & C & 1 & 2 & 3 & 4 & 5 \\
\hline $\mathrm{x}$ & & & & $x$ & & $\mathrm{X}$ & $X$ \\
\hline$X$ & & & & & & $\mathrm{x}$ & \\
\hline $\mathrm{X}$ & & & & & & $\mathrm{X}$ & \\
\hline $\mathrm{x}$ & & & & $\mathrm{X}$ & & & $\mathrm{X}$ \\
\hline $\mathrm{x}$ & & & & $\mathrm{X}$ & & & \\
\hline $\mathrm{x}$ & & & & $\mathrm{x}$ & & & \\
\hline & & $x$ & & & $\mathrm{X}$ & & \\
\hline $\mathrm{x}$ & & & & & $\mathrm{X}$ & & \\
\hline $\mathrm{x}$ & & & & & $\mathrm{X}$ & & \\
\hline $\mathrm{x}$ & & & & $x$ & & & \\
\hline $\mathrm{x}$ & & & & $\mathrm{x}$ & & & \\
\hline$x$ & $\mathrm{X}$ & $\mathrm{X}$ & & $\mathrm{x}$ & & & \\
\hline $\mathrm{X}$ & & & & & $\mathrm{x}$ & & \\
\hline & & $\mathrm{X}$ & $\mathrm{X}$ & & & & \\
\hline & & $\mathrm{X}$ & & $X$ & & & \\
\hline
\end{tabular}

$\mathrm{X}$

$x$

$\mathrm{x}$

$\mathrm{x}$

$\mathrm{X}$

$\mathrm{X}$

$\mathrm{X}$

X

$\mathrm{X}$

$\mathrm{X}$

$\mathrm{X}$

$\mathrm{X}$

$\mathrm{X}$

X

$\mathrm{X}$

$\mathrm{X}$

$\mathrm{X}$

$\mathrm{x}$

$\mathrm{X}$ semitectada

semitectada

semitectada

tectada perforada

microrretículada

tectada perforada

semitectada

fosulada

fosulada

semitectada

semitectada

tectada perforada

fosulada

tectada perforada

tectada perforada

a semitectada

tectada perforada

a semitectada

tectada perforada

semitectada

semitectada

intectada

semitectada

semitectada

semitectada

semitectada

semitectada

microrreticulada

semitectada

semitectada

semitectada

psilada

escabrada con foveolas

tectada perforada

tectada perforada

tectada perforada

tectada perforada semitectada

semitectada

semitectada

tectada perforada

microrreticulada

microrreticulada

semitectada

areolada

areolada

semitectada

semitectada

tectada perforada

microrreticulada

tectada perforada

tectada perforada

a semitectada

tectada perforada

tectada perforada semitectada

semitectada

intectada

semitectada

semitectada

semitectada

semitectada

semitectada

microrreticulada

microrreticulada

semitectada

semitectada

semitectada

puntitegilada

puntitegilada

puntitegilada

puntitegilada

puntitegilada

puntitegilada

Tipos de asociación del polen
A: eumónada
B: diáda
C: tétrada

Tipos de abertura

1, inaperturado. 2, monosulcado. 3, sulco tan largo como el eje ecuatorial mayor. 4, tricotomosulcado. 5, disulcado. 6, sulco en herradura. 7 , ulcerado. 8 , sulco con radio. 
CUADRO 7. Clasificación, distribución, características palinológicas, cariotipos y posición del ovario en Agavaceae.

Traub (1953)

\begin{tabular}{ll}
\hline Familia & Agavaceae \\
Tribu & Hosteae \\
& Hosta
\end{tabular}

Tribu

Tribu

Tribu

Tribu

Tribu

Tribu

Poliantheae

Polianthes

Prochnyanthes

Pseudobravoa

Agavoideae

Agave

Beschorneria

Furcraea

Doryanthes

Phormiaceae

Phormium

Dracaenaceae

Cordyline

Cohnia

Dracaena

Sansevieria

Familia

Dahlgren

et al., (1985)

Distribución

(Willis, 1980)

Orden Asparagales

Familia Funkiaceae

Hosta

Japón, China

Familia Agavaceae

Subfam. Yuccoideae

Hesperaloë

Yucca

E.U., México

E.U., México,

SO de E. U.

$S$ de E. U.,

México

Subfam. Agavoideae

Polianthes

Prochnyanthes México

México

Agave

$S$ de E.U. a

Sudamérica

Beschorneria México

Furcraea América

tropical

Manfreda

E.U. a El

Salvador

Doryanthaceae

Doryanthes Australia

Familia

Phormiaceae

Phormium

Nueva

Zelandia

Familia

Asteliaceae
Familia
Abertura

monosulcado

intectado

México monosulcado

(Verhoek, 1975)

(Verhoek, 1975)

(Verhoek, 1975)

Nolineae

Nolina

Dasylirion

Beaucarnea

Calibanus
Cordyline

Cohnia

Dracaenaceae

Dracaena

Sansevieria

Indomalasia, Nva.

Hawaii, Australia,

Africa, Sudamérica

(Dahlgren et al,

1985),

I. Mascareñas,

Nva. Caledonia

Africa subtropical, monosulcado

Asia hasta el $\mathrm{N}$ de

Australia

(Dahlgren et al.,

1985),

Africa subtropical, operculado

$\mathrm{S}$ de Madagascar a

Arabia

Subfam. Nolinaceae

Nolina

Dasylirion

$?$

?
Zelandia, Polinesia,
SO de E.U., monosulcado

México

SO de E.U., monosulcado

México

México-Honduras mono y

(Hernández,1993) disulcado

México monosulcado (Johnson y Gale, 1983) reticulado
Ornamentación

Cariotipo

(Gómez-Pompa

et al.,1971)

Ovario

monosulcado

monosulcado

mono, di y

tricotomosul-

cado

monosulcado

monosulcado

monosulcado

semitectado

tectado

perforado

$30(5 L+25 C)$

ínfero

ínfero

$30(5 \mathrm{~L}+25 \mathrm{C}) \quad$ ínfero

semitectado

semitectado

semitectado

semitectado

tricotomosul-

cado

semitectado

$24(2 \mathrm{~L}+22 \mathrm{C})$

ínfero

$16(4 \mathrm{~L}+12 \mathrm{C})$

súpero

$30(5 \mathrm{~L}+25 \mathrm{C}) \quad$ ínfero

(Verhoek, 1975)

súpero

súpero

súpero

$30(5 \mathrm{~L}+25 \mathrm{C})$
$30(5 \mathrm{~L}+25 \mathrm{C})$

$30(2 n-6 n)(5 L+25 C)$ ínfero

$30(5 \mathrm{~L}+25 \mathrm{C})$ ínfero

ínfero

remitectado

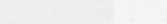

(n)

súpero

o


Calibanus y Beaucarnea como géneros válidos. El primero no pudo ser revisado en este estudio; sin embargo las observaciones palinológicas realizadas por Johnson y Gale (1983), sugieren que debe reconsiderarse su posición sistemática.
En una primera aproximación, Ojeda et al. (1984), al revisar seis géneros de la familia Agavaceae, concluyeron que con base en el polen resulta difícil mantener a la familia tal y como la propusieron Traub (1953) y Hutchinson (1934).

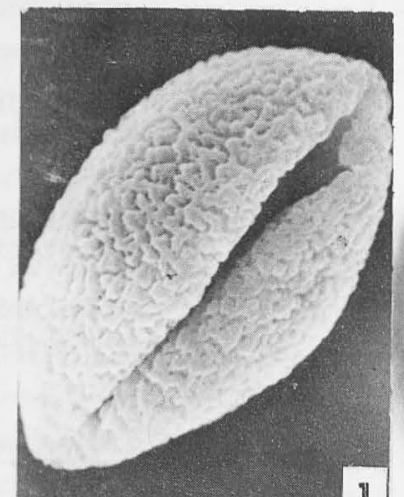

1

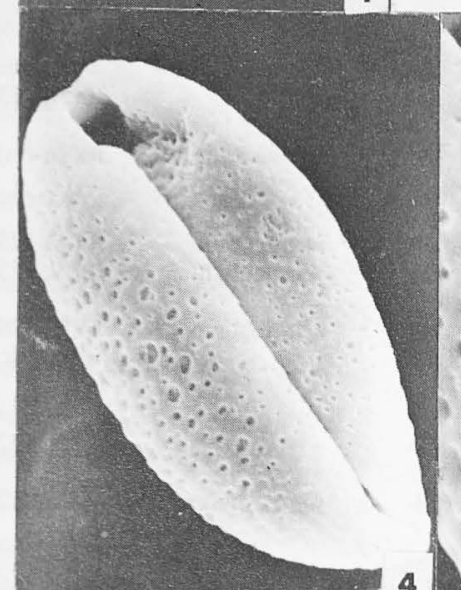

4

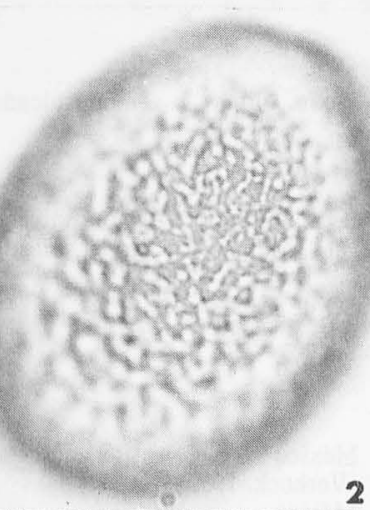

2

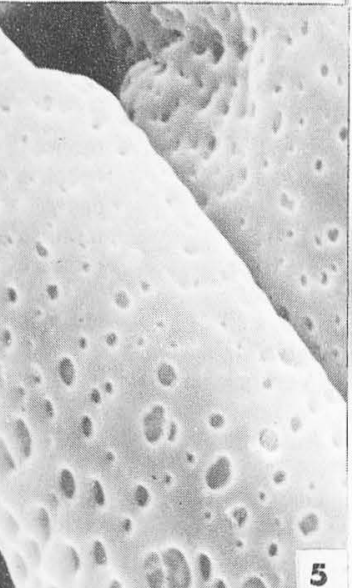

5

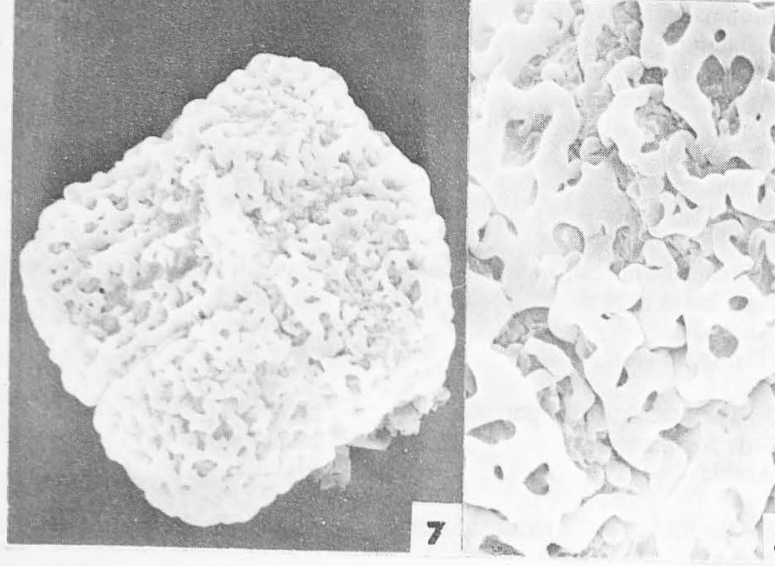

32

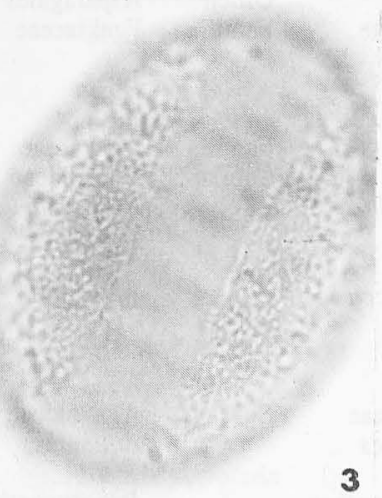

3

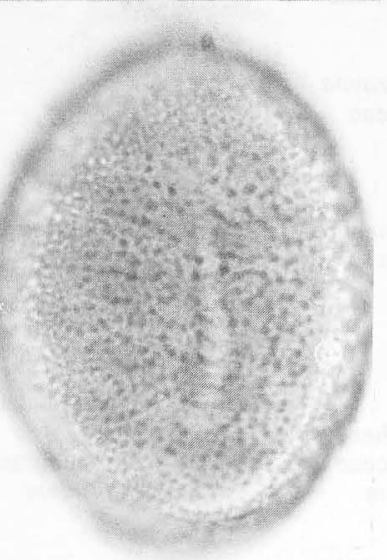

6

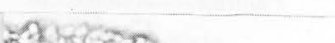

\section{(5)}

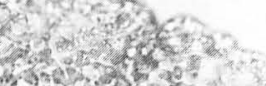

1.3.

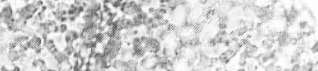

$x$ ton $^{2}$

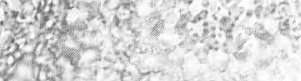

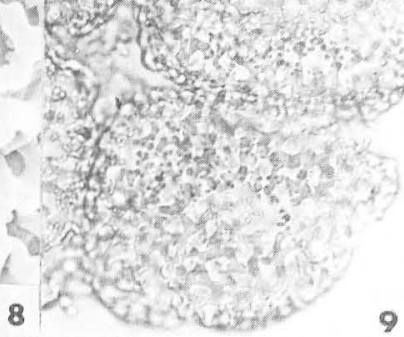

LÁmina I. Figs. 1 a 3, Beaucarnea inermis (Castillo 146 MEXU. 1 y 2, microrreticulado. 3. sulco tan largo como el eje ecuatorial mayor. Figs. 4 a 6, B. stricta (Trigos $529 \mathrm{MEXU}$ ). 4, terminaciones desiguales, microrreticulada hacia el polo proximal. 5 , exina en la zona de la abertura tectada perforada. 6, microrreticulada. Figs. 7 a 9. Berchorneria yuccoides (Hernández 4130 MEXU). 7, tétrada tetragonal. 8, exina semitectada, heterobrocada, muros discontinuos. 9, inaperturado, muros simpli a duplibaculados. 
La clasificación de Takhtajan (1980) es mas aproximada, ya que Dracaena, Cordyline y Sansevieria no presentan semejanzas con Agave, Yucca o Furcraea.

Al revisar los trabajos de Ludlow-Wiechers y Ojeda (1983) y Ojeda y Ludlow-Wiechers (1986), así como al integrar el polen de 10 géneros de la familia revisados en este trabajo, se reúne un total de 15 géneros y 36 especies. Aunque se observa una gran variación de tipos morfológicos, presentan algunas afinidades y patrones definidos (Cuadro 6).
Desde el punto de vista palinológico, las Agavaceae (sensu Traub, 1953) presentan dos niveles de comportamiento morfológico. A nivel interespecífico la variación encontrada es mínima o está ausente, lo cual da uniformidad a las especies que constituyen cada género. A nivel intergenérico, tomando en cuenta las aberturas y la ornamentación, se presentan los siguientes patrones morfológicos: (a) semitectado y principalmente monosulcado: Agave,
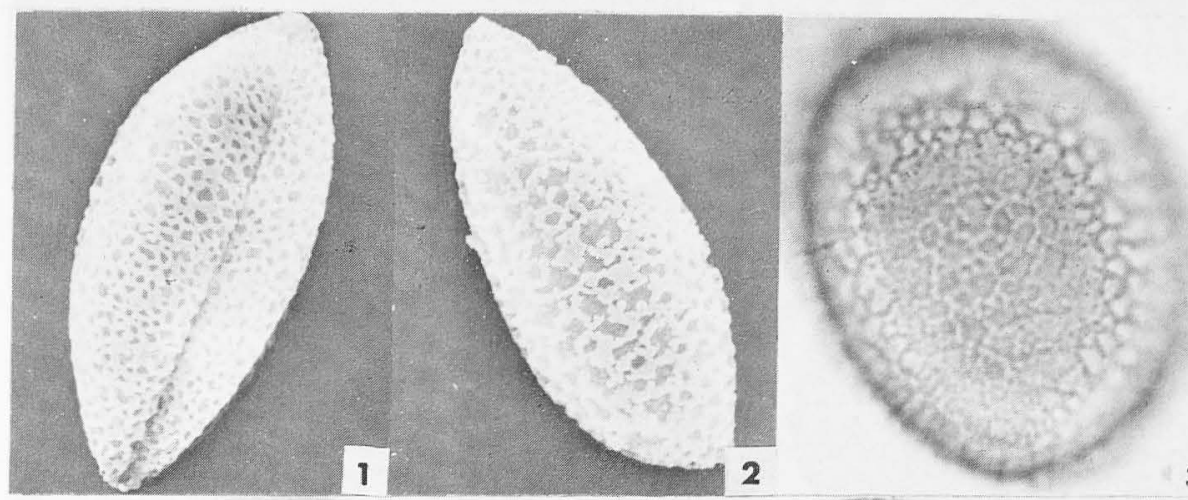

3

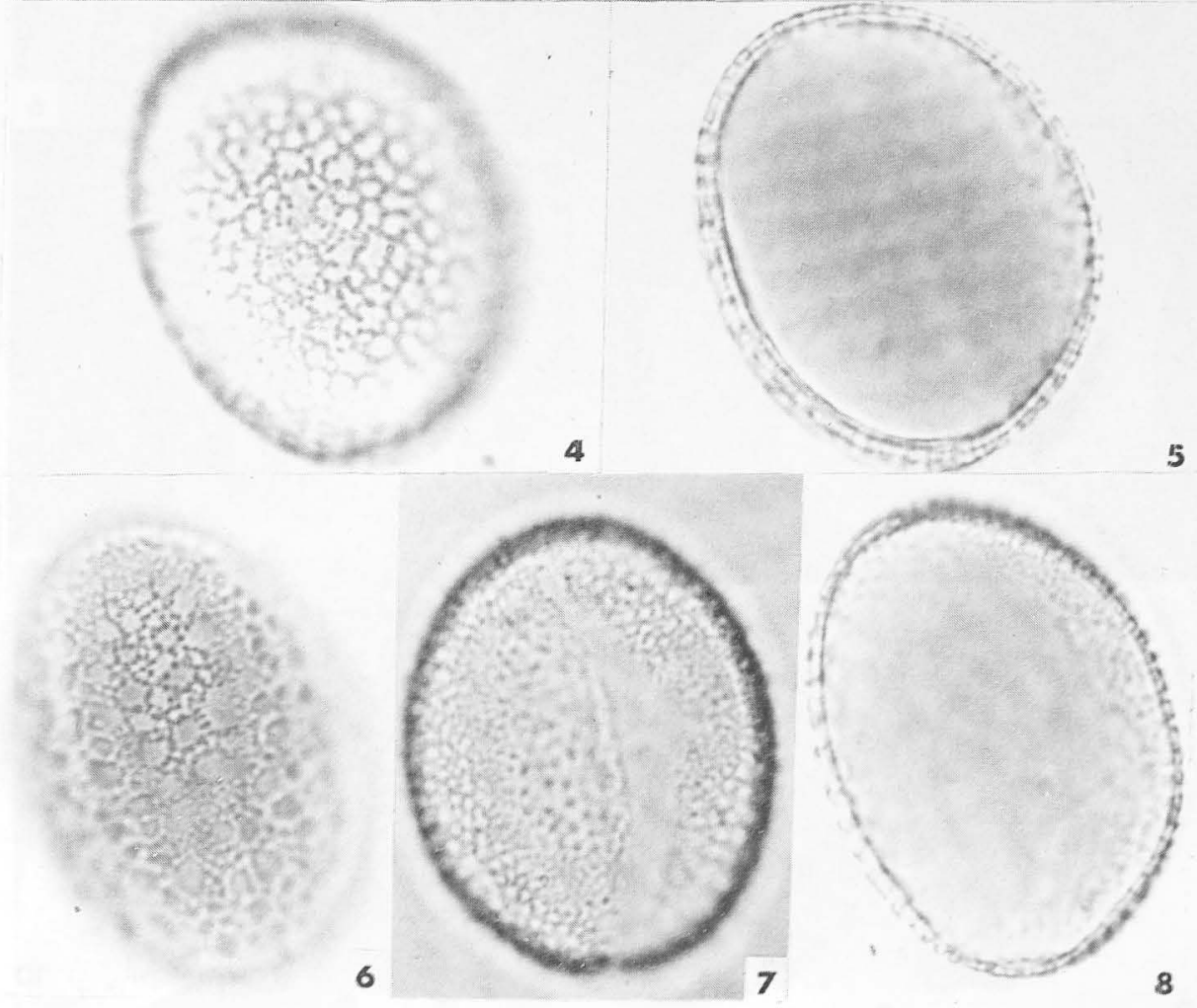


Beschorneria, Dasylirion, Furcraea, Hesperaloë, Manfreda y Polianthes; este último género a veces se presenta disulcado (Agavaceae, Agavoideae).

(b) Tectado perforado y monosulcado: Yucca (Agavaceae,
Yuccoideae) y Dracaena americana (Dracaenaceae).

(c) Tectado perforado a microrreticulado y monosulcado a disulcado: Beaucarnea y Nolina (Nolinaceae).

(d) Intectado verrugado y monosulcado: Hosta (Funkiaceae).

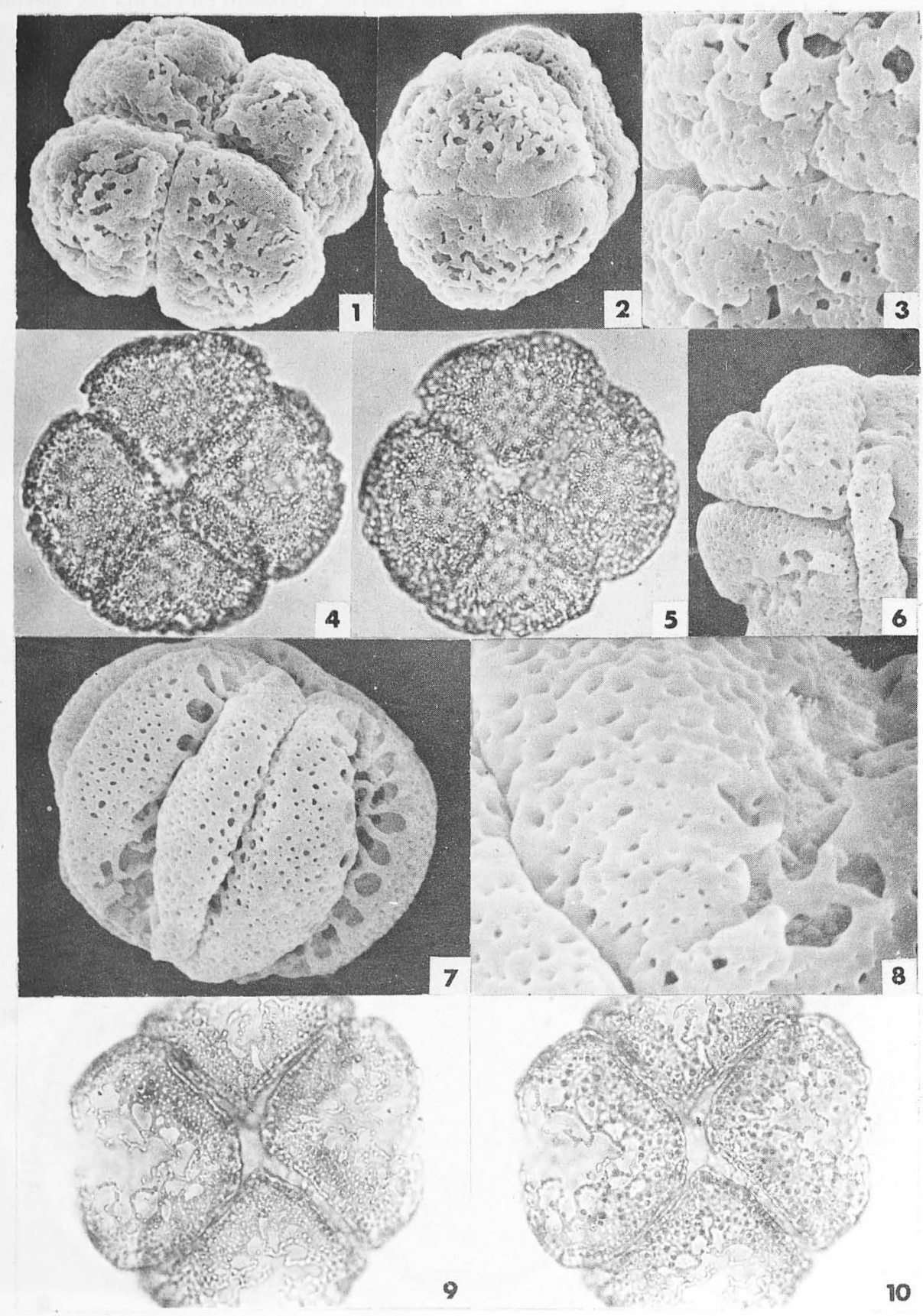

LÁmina III. Figs. 1 a 6, Furcraea bedinghausii (Matuda 37561 MEXU). 1, tétrada tetragonal, inaperturada. 2, semitectada en el polo distal. 3 , exina tectada perforada en la zona de contacto. 4, inaperturada. 5, simplibaculada. 6, acercamiento de una mónada. Figs. 7 a $10, F$. guatemalensis (Molina 26518 ENCB). 7, tétrada, exina semitectada a tectada perforada. 8, sulco con membrana y restos de la nexina. 9, tétrada teragonal, márgenes del sulco mal definidos, exina semitectada a tectada perforada, 10, simplibaculada. 
(e) Semitectado a tectado perforado y tricotomosulcado: Phormium (Phormiaceae).

(f) Fosulado y monosulcado: Cordyline (Asteliaceae) y Dracaena fragans (Dracaenaceae).

(g) Psilado y ulcerado: Sansevieria (Dracaenaceae).

Es necesario señalar que la asociación no tiene valor sistemático, y la agrupación en tétradas sólo señala una relación estrecha entre Furcraea y Beschorneria consideradas como pertenecientes a la subfamilia Agavoideae.

Al analizar detalladamente la situación de estos grupos (Cuadro 7), se observa que los géneros ubicados en el inciso (a) presentan una distribución netamente americana. Los géneros Agave, Beschorneria, Furcraea, Manfreda y Polianthes comparten además la posición ínfera del ovario y poseen un cariotipo similar $(n=30)$, por lo que
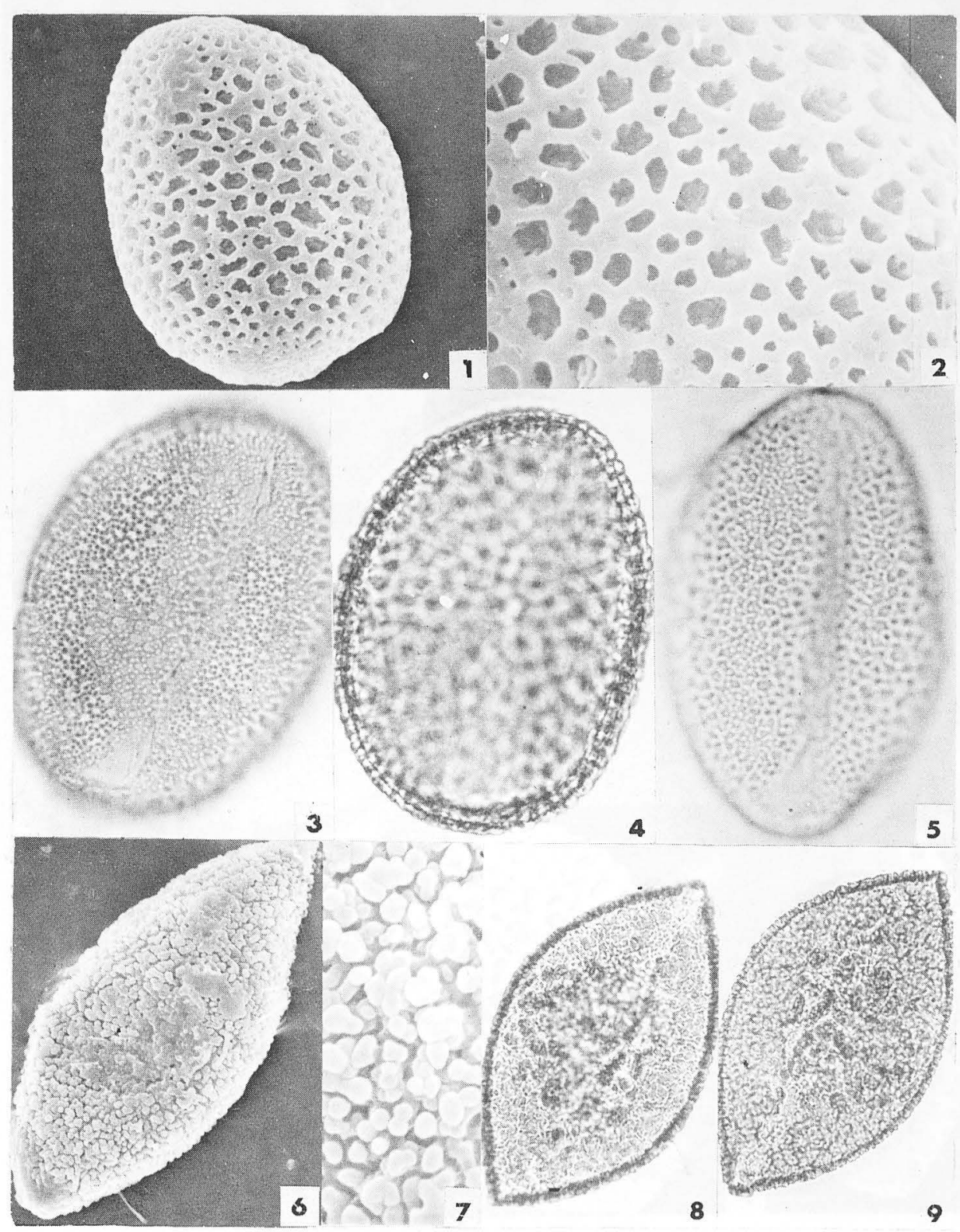

LÁmIna IV. Fig. 1, Hesperaloë funifera (Matuda 38791, ENCB). 1, exina semitecta. Figs. 2 a 5, H. parviflora (Johnston et al., 10892 MEXU), 2, exina semitectada. 3, sulco tan largo como el eje ecuatorial mayor, simpli a duplibaculada. 4, sección óptica. 5, sulco tan largo como el eje ecuatorial mayor. Figs. 6 a 9, Hosta sieboldiana (Yamazaki 7048 MEXU). 6, exina intectada. 7, verrugada a gemada. 8, monosulcada. 9, sección óptica, exina intectada. 
constituyen un grupo bien definido. El género Hesperaloë difiere de este grupo únicamente en la posición súpera del ovario, aunque su exina es más cercana a Agave, sin embargo, Dahlgren et al. (1985) loconsideran dentro de la tribu Yuccoideae, propuesta que coincide con la posición súpera del ovario, como lo presenta Yucca. Se puede proponer que Hesperaloë tuvo un origen común con Yucca y Agave, ya que presenta características comunes con ambos géneros (Cuadro 7). Dasylirion, por su
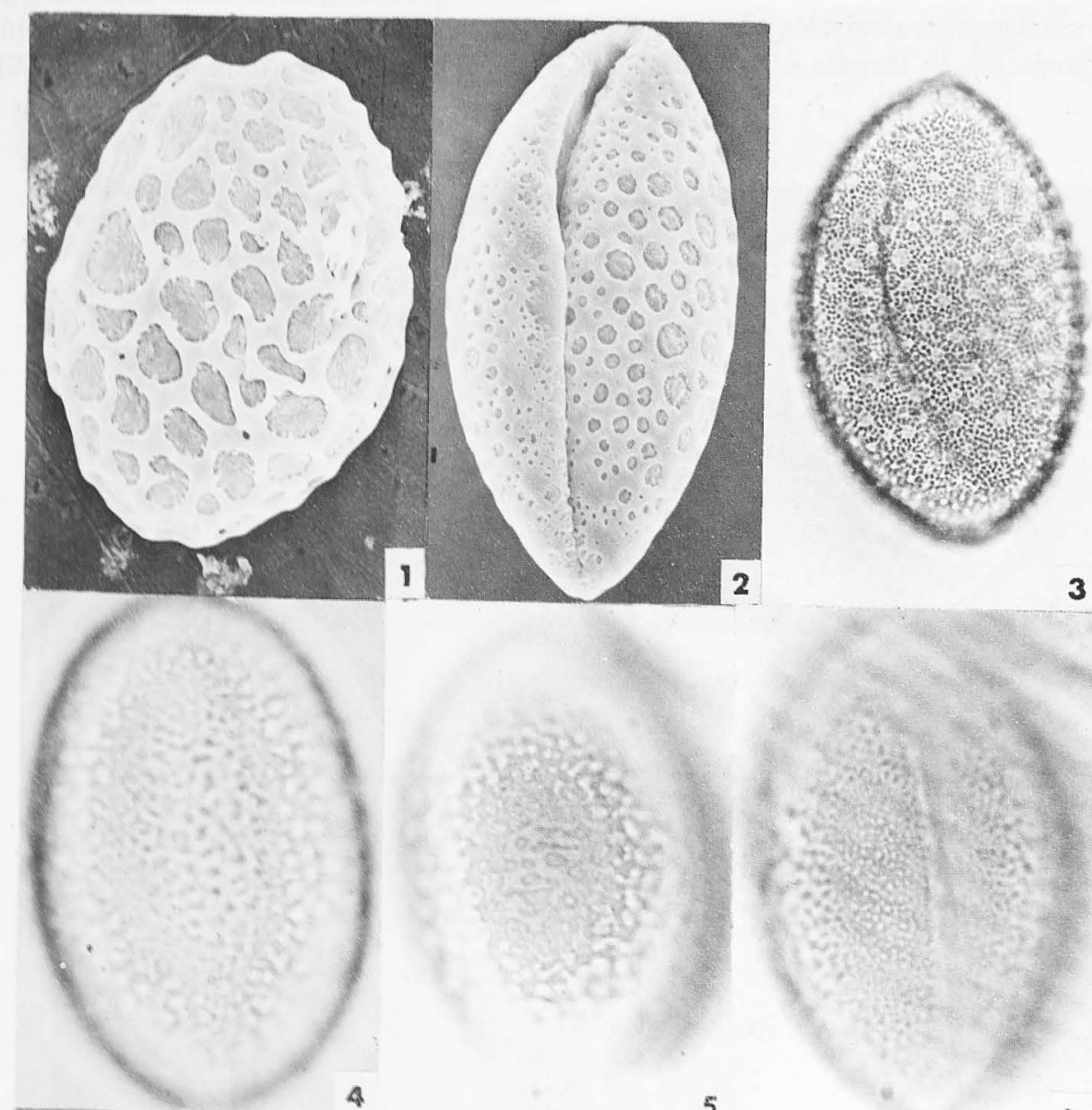

3

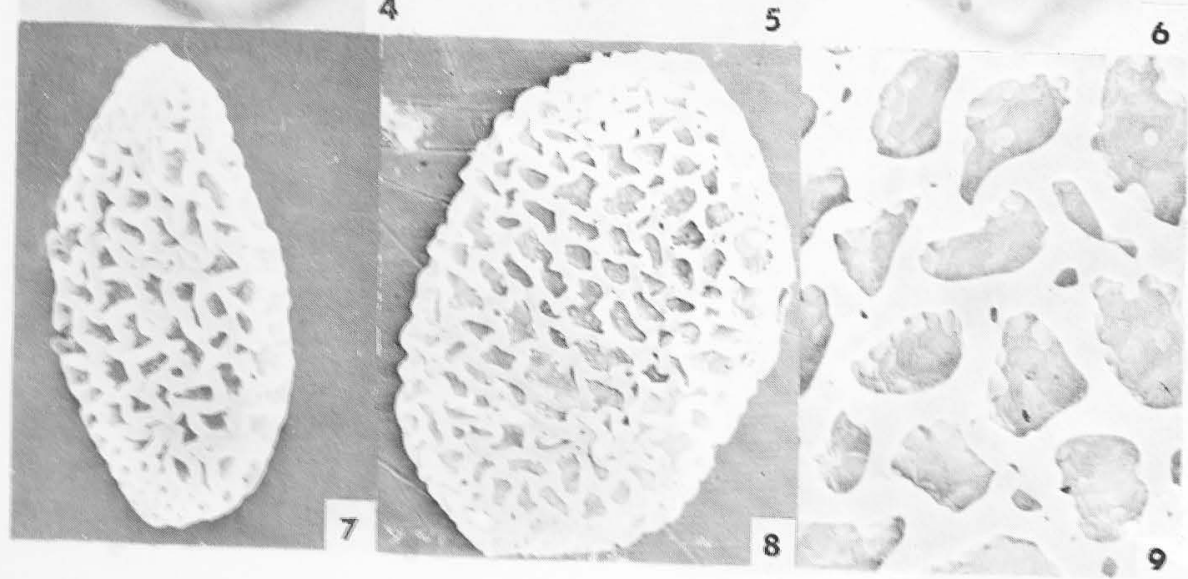

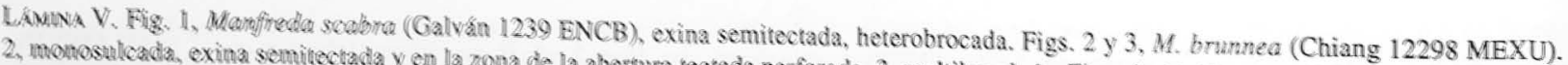

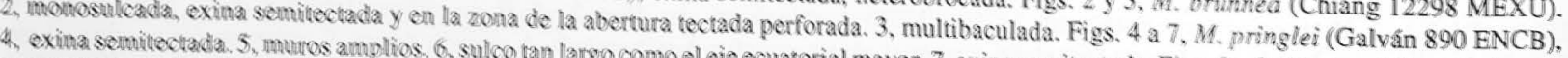
8542 MEXU). \&, extina semitectadia. 9, acercamiento. 
parte, posee un cariotipo muy diferente y la posición del ovario es súpera.

En las clasificaciones de Hutchinson (1964), Traub (1953) y Takhtajan (1980), la mayoría de estos géneros forman parte de la familia Agavaceae. Sin embargo, Manfreda no es considerado por estos autores y Polianthes no es tomado en cuenta por Takhtajan (1980). La única divergencia taxonómica de este grupo la constituye Dasylirion, el
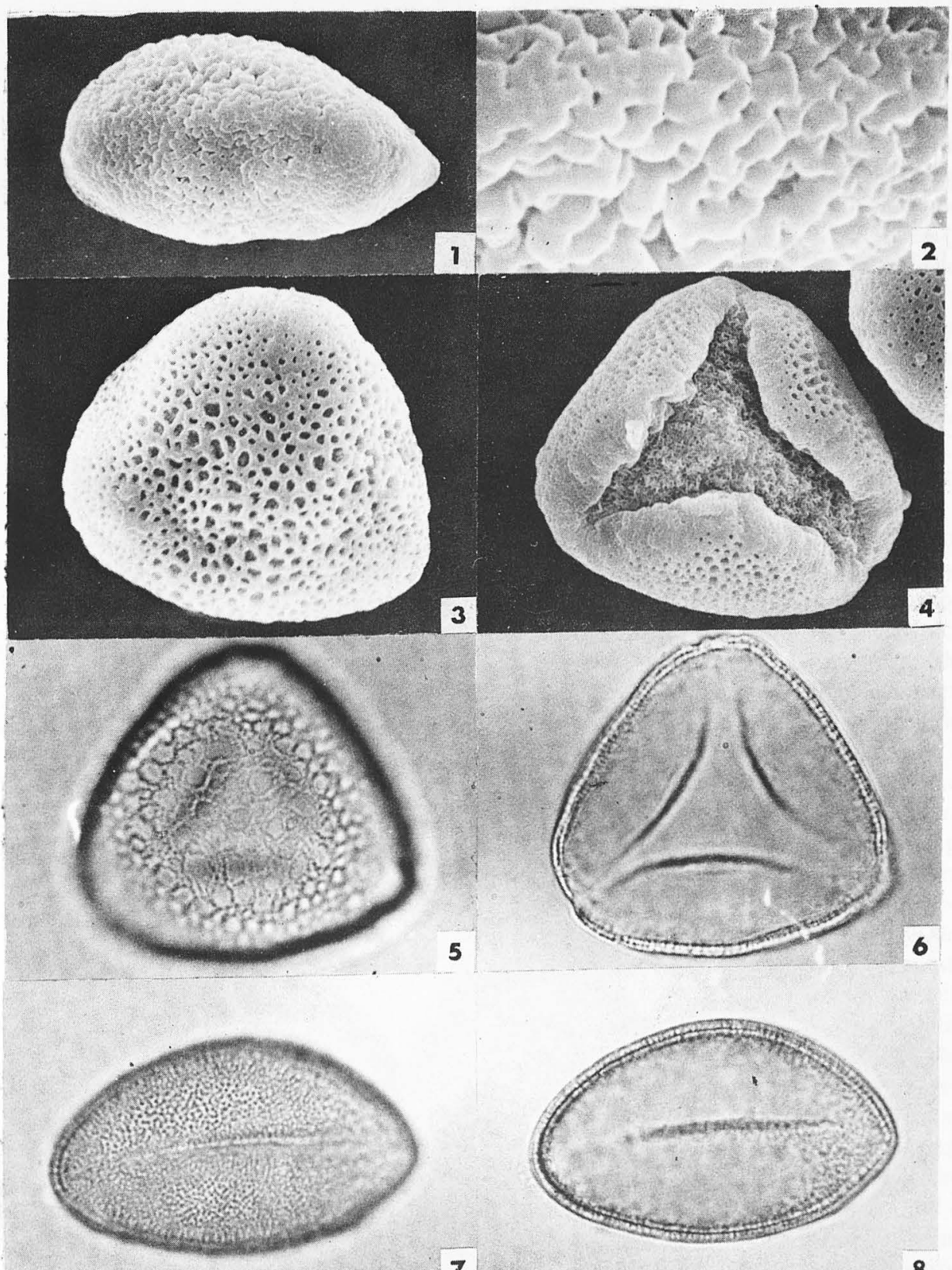

7

LÁmInA VI. Figs. 1 y 2, Nolina parviflora (Ramos 57 MEXU). 1, polo proximal, exina microrreticulada. 2, heterobrocada. Figs. 3 a 6, Phormium tenax (Koch 7621 MEXU). 3, exina semitectada. 4, tricotomosulcada, tectada perforada en la zona de la abertura, con membrana de la nexina muy evidente. 5, exina semitectada, simpli a duplibaculada. 6, sección óptica. Figs. 7 y 8, Yucca torreyi (Matuda 38560 ENCB). 7, ornamentación puntitegilada. 8 , sección óptica. 
cual es transferido por Takhtajan (1980) a otro suborden dentro de la familia Dracaenaceae. Por otra parte, Dahlgren et al. (1985) lo consideran dentro de la familia Nolinaceae. En estudios realizados sobre estos géneros Alvarez y Khöler (1987), encuentran que con base en la morfología del polen, se pueden agrupar cuatro subtipos: a) Agave-Manfreda, b) Manfreda-Prochnyanthes, c) Polianthes-Pseudobravoa y d) Hesperaloë.

Un segundo grupo está constituido por Yucca y Dracaena americana.Seconsideraque elgénero $Y$ ucca perteneceaAgavaceae
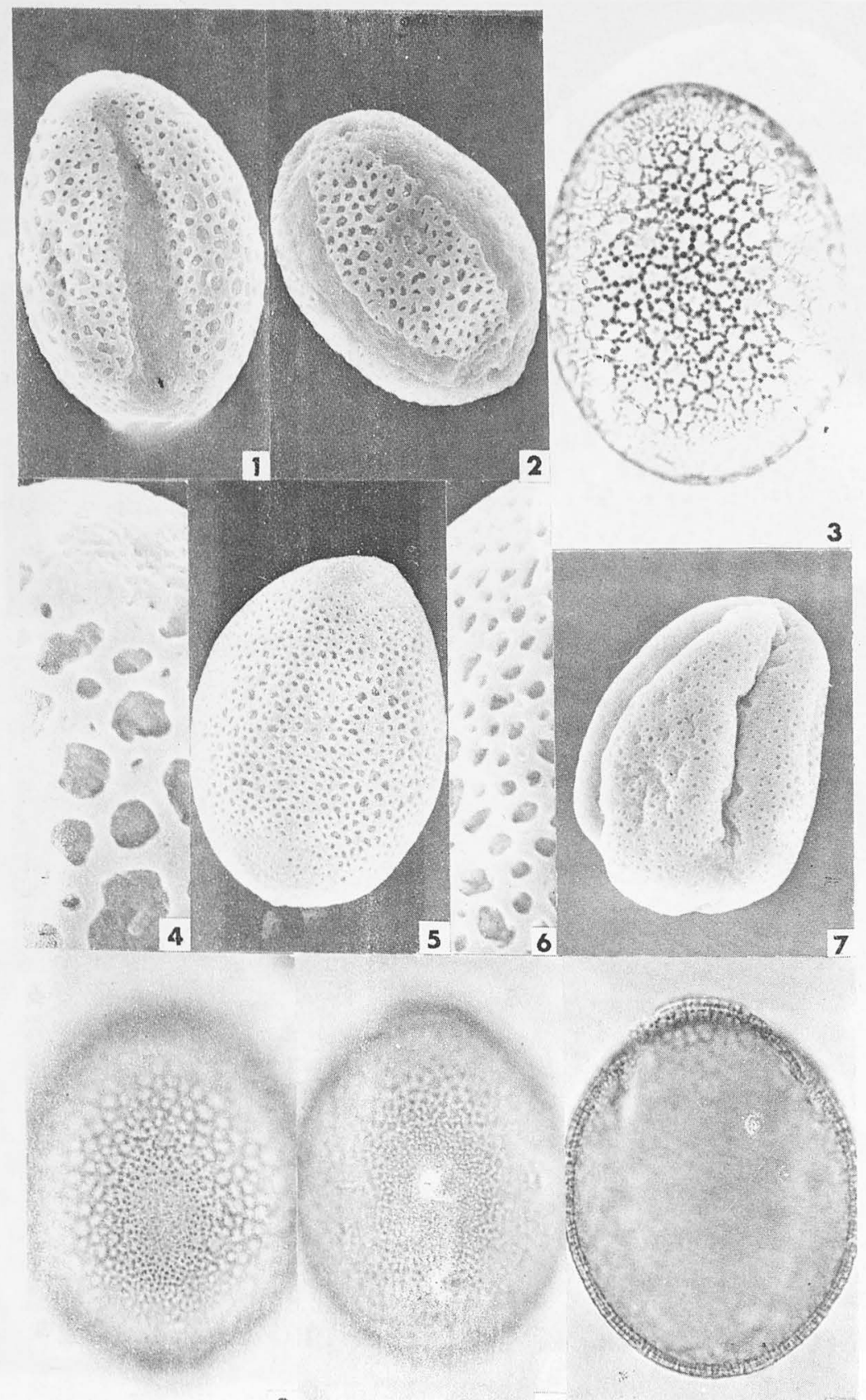

8

$9=$

10

Limins VII. Figs. 1 a 4, Polianthes gemin:flora (Hernánde $4556 \mathrm{MEXU}$ ). 1, monosulcada. 2, disulcada con membrana de la nexina en las aberturas. 3, semitectada, simplibaculada. 4, heterobrocada. Figs. 5 10, P. pringlei (Matuda 29250 MEXU). 5, exina semitectada. 6, heterobrocada. 7 , disulcada. 8, semitectada. 9, simplibaculada. 10 , sección 0 , ıca. 
dentro de la tribu Yucceae (Hutchinson, 1964; Traub, 1953; Takhtajan, 1980), sin embargo, Dahlgren y Clifford (1982) y Dahlgren etal.,(1985) losepara dentrodelasubfamilia Yuccoideae, ya que presenta el mismo cariotipo y distribución que los géneros de Agavoideae, pero la posición del ovario es súpera. En este estudio se encontró que, aunque el grano de polen presenta la misma abertura, la ornamentación de la exina varía presentándose tectada perforada. Dracaena americana será revisada mas adelante.

El tercer grupo esta constituido por Beaucarnea y Nolina, ambos tienen una distribución norteamericana, cariotipo similar $(\mathrm{n}=18-19)$ y ovario súpero. Beaucarnea, Nolina y Dasylirion son incluidos dentro de la tribu Nolineae, familia Agavaceae (Hutchinson, 1934; Traub, 1953). Gómez-Pompa et al. (1971) encuentran que el cariotipo de Dasylirion es muy similar al de Nolina y Beaucarnea. Takhtajan (1980) a pesar de no considerar al género Beaucarnea, une a Nolina y Dasylirion en la subfamilia Dracaenaceae. En cambio, Dahlgren et al. (1985) constituyen con estos géneros a la familia Nolinaceae. El polen de Dasylirion ha sido considerado muy semejante al polen de Agave; sin embargo, su cariotipo y la posición súpera del ovario lo apartan de la tribu Agavoideae, por lo que se propone incluirlo dentro de la familia Nolinaceae como lo hace Dahlgren et al. (1985). Los granos de polen de estos tres géneros tienen ornamentaciones que van de tectada perforada a microrreticulada a semitectada; además presentan variación en la zona cercana al sulco (Cuadro 7). Las observaciones de Johnson y Gale (1983) muestran que los granos de polen de Calibanus y Nolina con ornamentación tectada perforada a reticulada presentan una gran similitud entre ellos, mientras que el polen de Agave reconfirma las observaciones obtenidas en este trabajo, apoyadas por los estudios citológicos, ya que Calibanus presenta un cariotipo $(n=19)$. La propuesta de Johnson y Gale (1983) para considerar a Calibanus como un taxon válido, se ve apoyada por la posición intermedia que se presenta entre Beaucarnea y Nolina, ya que si bien el polen de Calibanus es muy semejante al de Nolina, su número cromosómico lo acerca más a Beaucarnea. Los géneros Beaucarnea, Calibanus, Dasylirion y Nolina presentan ovario súpero.

El género Hosta conforma el cuarto grupo. Es considerado por la mayoría de los autores (Hutchinson, 1934; Traub, 1953; Takhtajan, 1980) como parte de la familia Agavaceae ya que presenta similitud en su cariotipo $(\mathrm{n}=30)$ con el del grupo Yucca-Agave (Granick, 1944). Sin embargo, su distribución geográfica en China y Japón, la posición súpera del ovario y la morfología del polen sugieren que no tiene relación con los demás géneros estudiados, siendo el único con exina intectada, por lo que resulta difícil, considerarlo dentro de la familia Agavaceae. Dahlgren et al. (1985) lo incluyen dentro de la familia Funkiaceae (Cuadro 7).

En el quinto grupo, el género Phormium, con características especiales como su distribución geográfica confinada a Nueva Zelandia, ovario súpero, cariotipo $(n=16)$ y polen semitectado y tricotomosulcado, se diferencia notablemente del grupo de Agave. Aunque Hutchinson (1964) y Traub (1953) lo incluyen dentro de la familia Agavaceae, la clasificación de Takhtajan (1980) resulta más acertada, ya que lo separa y lo incluye dentro de la familia Phormiaceae, criterio que es sostenido por Dahlgren et al. (1985) (Cuadro 7).

Dentro del sexto grupo Dracaena y Cordyline presentan algunos problemas. Ambos poseen ovario súpero y cariotipo $(\mathrm{n}=19)$. En el género Cordyline se observan grandes diferencias en la morfología de polen con respecto a los demás géneros estudiados. En las dos especies de Dracaena estudiadas existen marcadas diferencias. Mientras que $D$. fragans presenta la misma abertura y ornamentación que Cordyline, $D$. americana es totalmente diferente. El género Dracaena es de origen africano con excepción de $D$. americana, presente en América, lo que puede confirmar lo propuesto por Ojeda y Ludlow-Wiechers (1985) de que este taxon amerita una revisión sistemática para definir su posición, ya que presenta gran relación con el polen tipo Yucca.

Los géneros Calibanus, Cordyline, Dasylirion, Dracaena, Nolina y Sansevieria que originalmente fueron incluidos por Hutchinson (1964) y Traub (1953) dentro de Agavaceae, son transferidos por Takhatjan (1980) a otro suborden dentro de la familia Dracaenaceae. Dahlgren et al. (1985) los separan en diferentes familias quedando Dracaena en Dracaenaceae y Cordyline en Asteliaceae (Cuadro 7).

Sansevieria, integrante del último grupo, presenta una distribución en África, Madagascar y Arabia, número cromosómico $(\mathrm{n}=20)$, ovario súpero y polen operculado, psilado a escabrado con foveolas. Hutchinson (1964) y Traub (1953) lo consideran en Agavaceae. Sharma y Chaudhuri (1964) proponen que el cariotipo de Sansevieria no justifica su inclusión, lo cual es apoyado posteriormente por Sen (1975). Takhtajan (1980) y Dahlgren et al. (1985) por su parte, proponen incluirlo en la familia Dracaenaceae. Desde el punto de visa del polen se justifica la transferencia fuera de Agavaceae ya que ninguna de las especies estudiadas presenta una ornamentación de la membrana tan distintiva y una úlcera tan desarrollada como la que posee Sansevieria. Sin embargo, la morfología del polen de Dracaeana fragans no presenta similitud con Sansevieria; quizás es necesario hacer una revisión de estos géneros para confirmar su posible separación dentro de la misma familia (Cuadro7).

Cabría señalar que el criterio palinológico para la delimitación de los géneros en Agavaceae ha sido considerado cuidadosamente, ya que la abertura monosulcada y la ornamentación semitectada se presentan en polen de Liliaceae y Amaryllidaceae; familias con características florales y vegetativas diferentes a los géneros aquí observados.

Suc (1975), en sus estudios palinológicos para el Plioceno de Languedoc, Francia, encontró cuatro granos de polen atribuibles a la familia Agavaceae, tres en tétradas y uno en mónada y sugiere que pueden ser Dracaena o Yucca. Si se comparan las fotografías de la tétrada con los resultados de Ojeda y Ludlow-Wiechers (1984), es posible sugerir que se trate de Dracaena, ya que ésta se ha encontrado en díadas y en tétradas. El grano de polen aislado se aproxima 
a Cordyline por la presencia de un sulco más largo que el eje ecuatorial mayor.

Como Dracaena y Cordyline se encuenteran distribuidas en las zonas tropicales y subtropicales del Viejo Mundo, es factible encontrarlas en un clima mediterráneo y que en el Plioceno tuvieron una distribución más amplia que la actual.

\section{AGRADECIMIENTOS}

Deseamos agradecer a la Biól. Lourdes Olivera, M. en C. Abisai García-Mendoza y Dra. Raquel Galván todo el amable apoyo recibido y sus acertadas sugerencias. Sin su colaboración, probablemente este trabajo no se hubiera publicado.

\section{LITERATURA CITADA}

Álvarez A, Köhler E. 1987. Morfología de las Agavaceae y algunos géneros afines. Grana 26: 25-45.

Bentham GD, Hooker JD. 1883. Genera Plantarum III. London, Reeve and $\mathrm{Co}$.

Berlin E. 1953. Yuccas y agaves de México. Memorias del Congreso Científico Mexicano 6: 362-367.

Bonefille MR. 1971. Atlas des pollens d'Ethiopie. Principales espèces des fôrets de montagne. Pollen et Spores 13: 15-72.

Conzatti C. 1946. Flora taxonómica mexicana. Tomo 2. México DF: Talleres Gráficos de la Nación.

Cranwell LM. 1953. New Zeland pollen studies. The monocotyledons. Bulletin of the Auckland Institute and Museum No. 3.

Cronquist A. 1968. The evolution and classification of flowering plants. Boston: Houghton Mifflin.

Dahlgren RMT, Clifford HT. 1982. The Monocotyledons. A comparative study. London: Academic Press.

Dahlgren RMT, Clifford HT, Yeo PF. 1985. The families of the Monocotyledons. Berlin: Springer-Verlag.

Erdtman G. 1943. An introduction to pollen analysis. New York: The Ronald Press Co.

Erdtman G.1952. Pollen morphology and plant taxonomy. Angiosperms. New York: Hafner.

García-Mendoza A. 1987. Monografía del género Beschorneria Kunth. Agavaceae. Tesis de Maestría. México DF: Facultad de Ciencias, UNAM.

García-Mendoza A. 1995. Riqueza y endemismos de la familia Agavaceae en México. En: Linares E, Dávila P, Chiang F, Bye R, Elias T. (eds.)Conservación de plantas en peligro de extinción: Diferentes enfoques. México DF: Instituto de Biología, UNAM.

Gómez-Pompa A, Villalobos-Pietrini R, Chimal A. 1971. Studies in the Agavaceae I. Chromosome morphology and number of seven species. Madroño 21: 208-221.

Gónzalez-Quintero L. 1969. Morfología Polínica: La flora del Valle de Mezquital, Hidalgo. Paleoecología 3. México DF: Instituto Nacional de Antropología e Historia.

Granick E. 1944. A karyosistematic study of the genus Agave. American Journal of Botany 31: 283-298.

Hernández SL. 1993. Cladistic analysis of the American genera of Asparagales and the systematic study of Beaucarnea (Nolinaceae) and Hemiphylacus (Hyacinthaceae). PhD thesis. Austin, USA:The University of Texas.

Heusser C. 1971. Pollen and spores of Chile. Tucson: The University of Arizona Press.

Howard RA. 1979. Agavaceae. En: (Eds.). Flora of Lesser Antilles. Vol III. Monocotyledonae. New York: Harvard University Press.
Huang. 1972. Pollenflora of Taiwan: Taiwan, National Taiwan University, Botany Department Press.

Hutchinson J. 1934. The families of flowering plants. Vol II. Monocotyledons. London: The Macmillan Co.

Hutchinson J. 1964. The families of flowering plants. Vol II. Monocotyledons. London: Oxford University Press.

Johnson MAT, Gale RMO. 1983. Observations on the leaf-anatomy, pollen, cytology and propagation of Calibanus hookeri (Lem.) Trelease. Bradleya 1: 25-32.

Kapp RO. 1969. How to know pollen and spores. USA: WMC Brown Company Publishers.

Kremp GOW. 1965. Morphological encyclopedia of Palynology. Tucson: The University of Arizona Press.

Ludlow-Wiechers B, Ojeda L. 1983. El polen del género Agave de la Península de Yucatán. Boletín de la Sociedad Botánica de México 44: 29-42.

Lundell CL. 1937. The Vegetation of Peten. Washington: Carnegie Institution. Apendix 1: Studies of Mexican and Central American plants.

Markgraff VH, D'Antoni H. 1978. Pollen flora of Argentina. Tucson: The University of Arizona Press.

Martínez-Hernández E. 1970. Estudios palinológicos de las especies dominantes de la vegetación de los alrededores de la Laguna Madre, Tamps. México. Tesis de Maestría. México DF: Facultad de Ciencias, UNAM.

Matuda E. 1960. Las Amarilidáceas y Liliáceas del Valle de México y sus alrededores. Anales del Instituto de Biología de México 31: $58-83$.

Matuda E. 1962. Las amarilidáceas del Estado de México. Toluca, México: Gobierno del Estado de México.

Matuda E, Piña LI. 1980. Las plantas mexicanas del género Yucca. México DF: Colección Misc., Edo. de México- Laboratorios Nacionales de Fomento Industrial..

McKelvey DS, Sax K. 1933. Taxonomic and cytological relatioship of Yucca and Agave. Journal of the Arnold Arboretum 14: 76-81.

McKelvey DS. 1938. Yuccas of the Southwestern United States. Part I. Jamaica Plain: Massachusetts: the Arnold Arboretum of Harvard University.

McKelvey DS. 1947. Yuccas of the Southwestern United States. Part II. Jamaica Plain: Massachusetts: the Arnold Arboretum of Harvard University.

Millspaugh CF. 1898. Contributions to the flora of Yucatan. Field Museum of Botany 4: 3-56.

Ojeda L, Ludlow-Wiechers B, Orellana R. 1984. Palinología de la familia Agavaceae para la Península de Yucatán. Biótica 9: 379-398.

Ojeda L, Ludlow-Wiechers B. 1986. Palinología de Agavaceae, una contribución biosistemática. Resúmenes del VI Coloquio sobre Paleobotánica y Palinología.

Palacios-Chávez R. 1974. Observaciones en el polen de plantas con probable polinización quiropterófila. Anales de la Escuela Nacional de Ciencias Biológicas. 21: 115-143.

Palacios-Chávez R. 1978. Morfología de los granos de polen de las especies mexicanas más comunes del género Yucca. Cactáceas y Suculentas Mexicanas 23: 3-8.

Palacios-Chávez R, Ludlow-Wiechers B, Ojeda L. 1988. Consideraciones sobre la exina en Yucca observadas al microscopio de luz y microscopio de barrido. Boletín de la Sociedad Botánica de México 48: 153-154.

Pax F, Hoffman K. 1930. Amaryllidaceae. En: Engler A. and Prantl K. (Eds.). Die Natürlichen Pflanzenfamilien. Leipzig: Verlag.

Praglowski J, Punt W, 1973. An elucidation of the microreticulate structure of exine. Grana 13: 45-50. 
Radulescu D. 1973. Liliflorae. Discussions et considèrations phylogenètiques a l'aide de quelques recherches morphopalinologiques. Acta Botanica Horti Bucarestiensis. 1972-1973: 249-283.

Satô R. 1935 Analysis of the caryotipes in Yucca, Agave and the related genera with special reference of the phylogenetic significance. Japananese Journal of Genetics 11: 273-278.

Sen S. 1975. Cytotaxonomy of Liliales. Feddes Reppertorium 86: 255 305.

Sharma AK, Battacharyya VC. 1962. A cytological study of the factors influencing evolution in Agave. La Cellule 62 (3): 259-280.

Sharma AK, Chaudhuri M. 1964. Cytological studies as an aid in assesing the status of Sansevieria, Ophiopogon and Curculigo. The Nucleus 7: 43-58.

Sharma AK. 1967. Pollen morphology of the Indian Monocotyledons. Journal Palynologycal. Lucknow. Special Volume.

Standley P. 1930. Flora of Yucatan. Field Museum History Botany Series 3: $157-429$.

Suc JP. 1975. Pollen attribuables aux Agavaceae dans le Pliocene du Languedoc. Pollen et Spores 16: 493-498.

Takhtajan PL. 1980. Outline of the classification of the flowering plants (Magnoliophyta). Botanical Review 46: 225-359.

Thorne RF. 1968. Synopsis of putative phylogenetic classification of the flowering plants. Aliso 6: 56-57.

Traub HP. 1953. The tribes and genera of the Agavaceae. Plant Life 9: 134-136.
Traub HP. 1972.Tribe Hosteae, family Agavaceae. Plant Life 28: 137-138.

Trelease W. 1911. The desert group Nolineae. Proceedings of the American Philosophical Society 50: 404-442.

Trelease W. 1920. Liliaceae, Amaryllidaceae. En: Standley PC. (Ed.). Trees and shrubs of Mexico. Contributions from the United States National Herbarium. 23: 87-142.

Tsukada M. 1964. Pollen morphology and identifications III. Modern and fossil tropical pollen. Pollen et Spores 6: 393-462.

Verhoek S. 1975. A study of the tribe Poliantheae (including Manfreda) and revisions of Manfreda and Prochnyanthes (Agavaceae). $\mathrm{PhD}$ thesis. Ithaca, New York: Cornell Unive sity.

Verhoek S. 1978. Huaco and amole. A survey of the uses of Manfreda and Prochnyanthes. Economic Botany 32: 124-130.

Vignoli L. 1936. Cariología del género Agave. Lavori Istituto Botánico Palermo 7: 176-217.

Vignoli L. 1937. Cariología del género Agave. Lavori Istituto Botánico Palermo 8: 1-15.

Whitaker JW.1934. Chromosome constitution in certain Monocotyledons. Journal of the Arnold Arboretum 15: 135-143.

Wiggins Y.1980. Flora of Baja California.Stanford: Stanford University Press.

Willis JC. 1980. A dictionary offlowering plants and ferns. Cambridge: Cambridge University Press.

Zavada M. 1983. Comparative morphology of monocot pollen and evolutionary trend of aperture and wall structures. Botanical Review 49: 331-379. 